\title{
Extensive White Matter Dysfunction in Cognitively Impaired Patients with Secondary-Progressive Multiple Sclerosis
}

\author{
P.L. Francis, T.L. Chia, R. Jakubovic, P. O'Connor, L. Lee, A. Feinstein, and R.I. Aviv
}

\begin{abstract}
BACKGROUND AND PURPOSE: Cognitive impairment is a common, disabling symptom of MS. We investigated the association between cognitive impairment and WM dysfunction in secondary-progressive multiple sclerosis using DTI.
\end{abstract}

MATERIALS AND METHODS: Cognitive performance was assessed with a standard neuropsychological battery, the Minimal Assessment of Cognitive Function in Multiple Sclerosis. Cognitive impairment was defined as scoring $>1.5$ standard deviations below healthy controls on $\geq 2$ subtests. Fractional anisotropy maps were compared against cognitive status using tract-based spatial statistics with threshold-free cluster enhancement.

RESULTS: Forty-five patients with secondary-progressive multiple sclerosis (median age: 55 years, female/male: 27/18, median Expanded Disability Status Scale Score: 6.5) were prospectively recruited. Cognitively impaired patients (25/45) displayed significantly less normalized global GM and WM volumes $(P=.001, P=.024)$, more normalized T2-weighted and T1-weighted WM lesion volumes $(P=.002, P=.006)$, and lower WM skeleton fractional anisotropy $(P<.001)$ than non-impaired patients. Impaired patients also had significantly lower fractional anisotropy $\left(p_{\text {corr }}<.05\right)$ in over $50 \%$ of voxels within every major WM tract. The most extensively impinged tracts were the left posterior thalamic radiation (100.0\%), corpus callosum (97.8\%), and right sagittal stratum (97.5\%). No WM voxels had significantly higher fractional anisotropy in patients with cognitive impairment compared with their non-impaired counterparts $\left(p_{\text {corr }}>.05\right)$. After the inclusion of confounders in a multivariate logistic regression, only fractional anisotropy remained a significant predictor of cognitive status.

CONCLUSIONS: Cognitively impaired patients with secondary-progressive multiple sclerosis exhibited extensive WM dysfunction, though preferential involvement of WM tracts associated with cognition, such as the corpus callosum, was apparent. Multivariate analysis revealed that only WM skeleton fractional anisotropy was a significant predictor of cognitive status.

ABBREVIATIONS: $C C=$ corpus callosum; EDSS = Expanded Disability Status Scale; FA = fractional anisotropy; MNI = Montreal Neurological Institute; RRMS = relapsing-remitting MS; SPMS = secondary-progressive MS; TBSS = tract-based spatial statistics; WML = white matter lesion

C ognitive impairment is a prevalent symptom of MS and has been estimated to occur in $43 \%-65 \%$ of patients. ${ }^{1}$ Those with secondary-progressive multiple sclerosis (SPMS) exhibit the highest frequency of impairment. ${ }^{2}$ Prior neuroimaging studies have almost exclusively investigated such impairment by assessing discrete cognitive domains, such as information processing speed or working memory, with neuropsychological tests that

Received July 24, 2013; accepted after revision March 18, 2014.

From the Departments of Psychiatry (A.F.), Neurology (L.L.), and Medical Imaging (P.L.F., T.L.C., R.J., R.I.A.), Sunnybrook Health Sciences Centre; Department of Neurology (P.O.C.), St. Michael's Hospital; and University of Toronto (P.O.C., L.L., A.F., R.I.A.), Toronto, Ontario, Canada.

This research was supported by the Physician Services Incorporated Foundation and the Multiple Sclerosis Scientific Research Foundation.

Please address correspondence to Philip L. Francis, MSc, Sunnybrook Health Sciences Centre, 2075 Bayview Ave, Room AB-202, Toronto, ON, M4N 3M5, Canada; e-mail: philip.francis@utoronto.ca

http://dx.doi.org/10.3174/ajnr.A3974 have established domain specificity. Such an approach is apparent in studies that examined both brain and lesion volumetry ${ }^{3-6}$ and WM integrity. ${ }^{7-11}$ Domain-specific cognitive tests are powerful tools that can assess the impact of MS pathology on individual cognitive domains and specific neuronal pathways, but cannot investigate cognition as a multidomain entity. Few studies of cognitive impairment in MS have adopted this broader perspective, and patients with MS with multiple domain impairment may exhibit considerably different structural brain damage from their counterparts with single domain impairment.

MS has been traditionally viewed as a disease predominantly affecting WM, though only modest associations between T2weighted hyperintense or T1-weighted hypointense white matter lesion (WML) load and cognitive test performance have been reported. ${ }^{12}$ Normal-appearing white matter is being increasingly examined, particularly through DTI techniques..$^{7-11,13,14}$ These studies have most frequently used fractional anisotropy (FA) as 
their primary outcome, ${ }^{8-11,13,14}$ whereas mean diffusivity has been investigated to a lesser extent. ${ }^{7}$ Radial diffusivity and axial diffusivity have been examined as secondary outcomes. ${ }^{1,14}$ Given the recent emphasis on the role of GM pathology both in the etiology of MS and related cognitive dysfunction, FA was considered in the context of GM volume using multivariate regression. ${ }^{15-17}$ Normal-appearing white matter has also been investigated using magnetization transfer ratio. ${ }^{18}$

Tract-based spatial statistics (TBSS) is being increasingly utilized in DTI studies of MS and cognition. ${ }^{8,9,11,13}$ TBSS has improved on traditional voxel-based morphometry by thinning WM to invariant tracts common to all patients. ${ }^{19}$ The voxel-based morphometry approach suffers from deficiencies related to spatial alignment and smoothing, which are mitigated by TBSS. Several prior TBSS studies have concluded that cognitive impairment in MS is because of the selective disruption of specific WM tracts associated with cognition, such as the cingulum and corpus callosum (CC). ${ }^{8,9,11}$ It should be noted that this spatial specificity has a physiologic basis and is not mediated by the cognitive nature of these tracts. These studies correlated FA values with performance on individual cognitive tests and examined cohorts primarily or solely comprising patients with relapsing-remitting MS (RRMS). DTI outcomes in patients with SPMS with and without cognitive impairment have not been well investigated, yet $65 \%$ of patients with RRMS will progress to SPMS. ${ }^{20}$ While WM injury is initially selective for specific tracts associated with impaired cognition in RRMS, DTI abnormalities are expected to become significantly more widespread and generalized with greater impairment and disease severity progression.

We hypothesized that patients with SPMS with impairment spanning multiple cognitive domains should exhibit extensive WM dysfunction not restricted to tracts implicated in cognition, such as the cingulum and CC.

\section{MATERIALS AND METHODS \\ Patients}

This study was approved by the research ethics boards of Sunnybrook Health Sciences Centre and St. Michael's Hospital. Patients with SPMS were prospectively recruited during a 1-year period from 2 tertiary referral MS clinics. SPMS diagnosis was based on the opinion of a senior neurologist with specialist practice in MS (20 years' experience). Charts of potential participants were screened by the same senior neurologist before recruitment to ensure eligibility. Exclusion criteria were: history of drug/alcohol abuse, use of disease-modifying drugs or steroids within the past 6 months, premorbid (ie, pre-MS) psychiatric history, head injury with loss of consciousness, concurrent medical diseases (eg, cerebrovascular disease), and contraindication to MR imaging. Clinical data included: age, sex, education level, and disease duration. MR imaging acquisition, neurologic examination, and Expanded Disability Status Scale (EDSS) assessment ${ }^{21}$ were completed on the same day.

\section{Cognitive Testing}

The Minimal Assessment of Cognitive Function in Multiple Sclerosis was administered under the supervision of a senior neuropsychiatrist. $^{22}$ This standard MS cognitive battery is a compre- hensive assessment tool consisting of 7 neuropsychological tests: Paced Auditory Serial Addition Test (working memory), Symbol Digit Modalities Test (processing speed), California Verbal Learning Test, 2nd Edition (verbal memory), Brief Visuospatial Memory Test, Revised (visuospatial memory), Delis-Kaplan Executive Function System (executive function), Controlled Word Association Test (verbal fluency), and Judgement of Line Orientation (visuospatial perception). Impairment on an individual test was defined as scoring more than 1.5 standard deviations below normative data of healthy controls. ${ }^{23}$ Patients with 2 or more test impairments were designated as having cognitive impairment. ${ }^{23}$ Beck Depression Inventory scores were also obtained because of the association between depression and cognitive impairment in patients with MS. ${ }^{4}$

\section{MR Imaging Acquisition}

MR imaging scanning was performed on a 3T scanner (Philips Healthcare, Best, the Netherlands) with a 16-channel phased array coil. The following sequences were acquired: 1 ) T1 3D (TR/ TE: $9.5 \mathrm{~ms} / 2.3 \mathrm{~ms}$, resolution: $\left.0.71 \times 0.71 \times 1.4 \mathrm{~mm}^{3}\right)$; 2 ) proton density/T2 (TR/TE: $2900 \mathrm{~ms} / 10.7 \mathrm{~ms}$, resolution: $0.45 \times 0.45 \times 3$ $\mathrm{mm}^{3}$ ); 3) DTI (TR/TE: $8966 \mathrm{~ms} / 57 \mathrm{~ms}$, resolution: $2.95 \times 2.95 \times$ $3 \mathrm{~mm}^{3}$, 50 sections, b-value: 1000 seconds $/ \mathrm{mm}^{2}$, single $\mathrm{b}=0$ scan, 32 gradient directions, 2 sequential scans [co-registered and averaged off-line]).

\section{Image Processing}

The structural scans were processed to generate global GM, WM, T2-weighted WML, and T1-weighted WML tissue volumes normalized to total intracranial volume as previously reported. ${ }^{17}$ Briefly, regions of GM and WM were delineated algorithmically, and T2-weighted hyperintensities and T1-weighted hypointensities were hand traced using Medical Image Processing, Analysis, and Visualization version 4.0 (National Institutes of Health, Bethesda, Maryland). The FMRIB Software Library (FSL, http:// www.fmrib.ox.ac.uk/fsl) processed the DTI scans. ${ }^{24}$ The FSL FLIRT tool co-registered the 2 sequential DTI scans per patient by using a 12-parameter affine transform, and the co-registered scans were averaged using Fslutils (http://fsl.fmrib.ox.ac.uk/fsl/ fslwiki/Fslutils). This approach avoids averaging on the scanner, which is inappropriate because of EPI susceptibility artifacts. ${ }^{25}$ The FSL Diffusion Toolbox corrected the co-registered, averaged scans for motion and eddy current distortions before fitting the diffusion tensors and calculating the FA maps. The acquisition space FA maps were normalized to Montreal Neurological Institute (MNI) space using the FSL FNIRT tool. The mean MNI space FA map for all patients was computed and thinned to invariant WM tracts common to every patient. This mean MNI space FA skeleton was thresholded at 0.2 before being applied as a mask to skeletonise the MNI space FA map for each individual patient to exclude non-WM voxels.

\section{Statistical and Image Analysis}

Clinical data were compared between patients with and without cognitive impairment using the Wilcoxon rank sum test for continuous variables or Pearson $\chi^{2}$ test for dichotomous variables. Results for continuous variables were expressed as median (inter- 


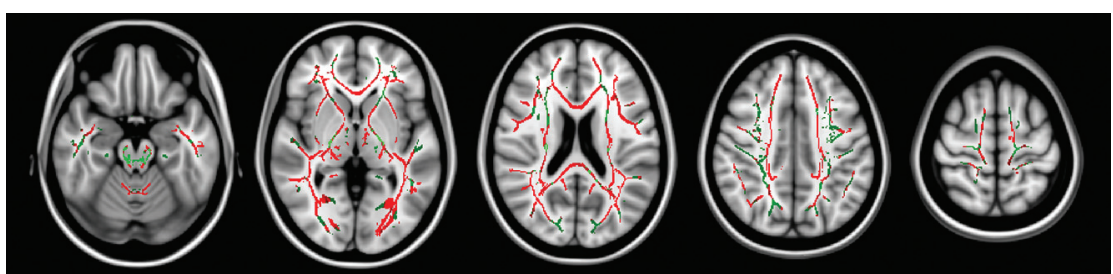

FIG 1. Significantly reduced WM FA values in cognitively impaired patients with SPMS. Inferior to superior arrangement of axial sections with 2 different color overlays. The red overlay represents voxels of significantly reduced FA in cognitively impaired patients with SPMS within the WM skeleton. The green overlay represents the remainder of the WM skeleton where no such significant association was identified.

Table 1: Clinical and radiologic characteristics of patients with SPMS

\begin{tabular}{lccr}
\hline & $\begin{array}{c}\text { Without Cognitive } \\
\text { Impairment } \\
(\boldsymbol{n}=\mathbf{2 0})\end{array}$ & $\begin{array}{c}\text { With Cognitive } \\
\text { Impairment } \\
(\boldsymbol{n}=\mathbf{2 5})\end{array}$ & $\boldsymbol{P}$ Value \\
\hline Age (y) & $54.5(49.3-62.3)$ & $55.0(47.5-61.5)$ & $.954^{\mathrm{a}}$ \\
Sex (F/M) & $11 / 9$ & $16 / 9$ & $.540^{\mathrm{b}}$ \\
Education level (y) & $15.5(13.0-17.8)$ & $14.0(13.0-17.0)$ & $.619^{\mathrm{a}}$ \\
Disease duration (y) & $18.5(10.5-20.8)$ & $19.0(12.0-28.5)$ & $.192^{\mathrm{a}}$ \\
EDSS (score) & $6.0(6.0-6.5)$ & $6.5(6.0-6.5)$ & $.249^{\mathrm{a}}$ \\
BDI (score) & $6.5(5.0-11.8)$ & $9.0(7.5-13.5)$ & $.067^{\mathrm{a}}$ \\
Normalized GM volume (\%) & $41.8(41.5-42.9)$ & $40.5(38.7-41.6)$ & $.001^{\mathrm{a}}$ \\
Normalized WM volume (\%) & $34.2(33.9-35.1)$ & $33.8(33.3-34.3)$ & $.024^{\mathrm{a}}$ \\
Normalized T2-weighted WML volume (\%) & $0.40(0.08-0.94)$ & $1.05(0.63-1.78)$ & $.002^{\mathrm{a}}$ \\
Normalized T-weighted WML volume (\%) & $0.12(0.03-0.32)$ & $0.44(0.13-0.67)$ & $.006^{\mathrm{a}}$ \\
WM skeleton FA (mean) & $0.37(0.34-0.38)$ & $0.33(0.28-0.34)$ & $<.001^{\mathrm{a}}$ \\
\hline Note & &
\end{tabular}

Note:- EBSS indicates Expanded Disability Status Scale; BDI, Beck Depression Inventory.

${ }^{a}$ Wilcoxon rank-sum test (median [interquartile range]).

b Pearson $\chi^{2}$ test.

quartile range), and results for dichotomous variables were expressed as proportions.

The normalized global tissue volumes were analyzed with respect to cognitive status as previously described. ${ }^{17}$ The skeletonized FA maps were compared between patients with and without cognitive impairment using FSL's Randomise tool, which conducts permutation-based nonparametric testing while correcting for multiple comparisons across space. ${ }^{26}$ To be conservative and assumption free, voxelwise group analysis was conducted with 5000 permutations and threshold-free cluster enhancement $\left(p_{\text {corr }}<.05\right)$. Threshold-free cluster enhancement is a recent advance in TBSS methodology that eliminates the need for an arbitrarily chosen cluster size threshold. ${ }^{27}$ The results appeared as images with voxel values consisting of corrected $P$ values. The WM parcellation map based on the MNI space ICBM-DTI-81 atlas provides stereotaxic delineation of every major WM tract in the brain and was employed as an region of interest template. ${ }^{28}$ This WM tract region of interest template was applied as an overlay on the results images, and both the total voxel count and number of significant voxels were algorithmically extracted for every region of interest. ROIs found to contain less than 100 voxels when superimposed on the mean FA skeleton were excluded from subsequent analyses. A list of included ROIs is provided in Table 2. Mean FA values for the WM skeleton of each patient were also calculated. A multivariate logistic regression model was used to identify factors predictive of cognitive status. Putative factors submitted to multivariate analysis were those demonstrating a $P<.1$ association with cognition in univariate analysis.

\section{RESULTS}

Clinical and Radiologic

Characteristics

Of 45 patients with SPMS prospectively recruited, 25 (55.6\%) were classified as being cognitively impaired. The clinical characteristics, including age, sex, education level, disease duration, and EDSS score, were not significantly different between patients with and without cognitive impairment $(P>.05)$ (Table 1). There was a trend toward higher Beck Depression Inventory scores in impaired patients, but this did not reach clinical significance $(P=.07)$. Impaired patients displayed significantly reduced normalized global GM volume $(P=.001)$, significantly lower normalized global WM volume ( $P=.024)$, significantly higher normalized T2-weighted WML volume $(P=.002)$, significantly greater normalized T1-weighted WML volume $(P=$ .006), and significantly lower WM skeleton FA $(P<.001)$ than patients without cognitive impairment. No significant difference in normalized global WM volume was found $(P>$ .05) (Table 1). Following multivariate analysis, WM skeleton FA was the only factor that remained a significant predictor of cognitive status $(P=.03)$.

\section{WM Integrity and Cognition}

Patients with cognitive dysfunction had significantly lower FA values in over $50 \%$ of the voxels within every major WM tract compared with those without $\left(p_{\text {corr }}<.05\right)$ (Fig 1, Table 2). The most extensively affected WM tracts were the left posterior thalamic radiation $(100.0 \%)$, CC $(97.8 \%)$, right sagittal stratum (includes inferior fronto-occipital fasciculus and inferior longitudinal fasciculus, 97.5\%), right posterior thalamic radiation (96.1\%), and left sagittal stratum (95.4\%). Only 1 other region (right medial lemniscus) had greater than $90 \%$ alteration (90.8\%). These findings illustrate that although WM dysfunction is predominantly generalized with respect to cognitive status, preferential involvement of specific WM tracts was apparent. No WM voxels had significantly higher FA in patients with cognitive impairment compared with their nonimpaired counterparts $\left(p_{\text {corr }}>.05\right)$.

\section{DISCUSSION}

Cognitively impaired patients with SPMS in the present series exhibited significantly reduced normalized global GM and WM volumes, significantly increased normalized T2-weighted and T1weighted WML volumes, and significantly lower WM skeleton FA consistent with the assertion that cognitive impairment is associated with more advanced disease. Multivariate analysis of factors predicting cognitive status demonstrated that only WM skeleton FA was significant. Our results demonstrate that in advanced MS 
Table 2: Tract-based spatial statistics group analysis identifying WM tracts with significantly lower FA values in patients with cognitive impairment compared with patients without cognitive impairment

\begin{tabular}{lrcc}
\hline & $\begin{array}{c}\text { Total } \\
\text { Voxels }\end{array}$ & $\begin{array}{c}\text { Significant } \\
\text { Voxels }\end{array}$ & $\begin{array}{c}\% \\
\text { Significant }\end{array}$ \\
\hline Middle cerebellar peduncle & 2608 & 2068 & $79.3 \%$ \\
Corpus callosum & 7044 & 6891 & $97.8 \%^{\mathrm{a}}$ \\
R corticospinal tract & 134 & 72 & $53.7 \%$ \\
L corticospinal tract & 208 & 154 & $74.0 \%$ \\
R medial lemniscus & 174 & 158 & $90.8 \%$ \\
L medial lemniscus & 209 & 171 & $81.8 \%$ \\
R cerebellar peduncle & 476 & 331 & $69.5 \%$ \\
L cerebellar peduncle & 414 & 294 & $71.0 \%$ \\
R cerebral peduncle & 575 & 502 & $87.3 \%$ \\
L cerebral peduncle & 600 & 415 & $69.2 \%$ \\
R internal capsule & 2219 & 1728 & $77.9 \%$ \\
L internal capsule & 1659 & 1308 & $78.8 \%$ \\
R corona radiata & 3935 & 3267 & $83.0 \%$ \\
L corona radiata & 3142 & 2486 & $79.1 \%$ \\
R posterior thalamic radiation & 1086 & 1044 & $96.1 \%{ }^{\mathrm{a}}$ \\
L posterior thalamic radiation & 554 & 554 & $100.0 \%{ }^{\mathrm{a}}$ \\
R sagittal stratum & 652 & 636 & $97.5 \%{ }^{\mathrm{a}}$ \\
L sagittal stratum & 345 & 329 & $95.4 \%$ \\
R external capsule & 1115 & 993 & $89.1 \%$ \\
L external capsule & 565 & 437 & $77.3 \%$ \\
R cingulum & 526 & 397 & $75.5 \%$ \\
L cingulum & 319 & 220 & $69.0 \%$ \\
R superior longitudinal fasciculus & 1614 & 1363 & $84.4 \%$ \\
L superior longitudinal fasciculus & 1338 & 840 & $62.8 \%$ \\
\hline Not & & &
\end{tabular}

Note:-R indicates right; L, left.[br /]

a Regions with greater than 95\% WM impingement.

disease characterized by SPMS, multiple domain cognitive dysfunction is the result of diffuse WM injury with regional predilections present in the $\mathrm{CC}$, posterior thalamic radiation, and sagittal stratum. Of these 3 regions only the $\mathrm{CC}$ is traditionally associated with cognition, ${ }^{29}$ though a recent study implicated the posterior thalamic radiation in intellectual performance. ${ }^{30}$ Thalamocortical circuit integrity has additionally been found to differentiate normal aging from mild cognitive impairment. ${ }^{31}$ This suggests that thalamic radiations are implicated in a wide range of cognitive tasks. Furthermore, significantly reduced FA was recently reported in the inferior longitudinal fasciculus, a component of the sagittal stratum, of patients with mild cognitive impairment. ${ }^{32}$

The observed differences in the spatial extent of WM dysfunction between the present SPMS study and those investigating RRMS allow for inference regarding the interplay of disease progression, WM integrity, and cognitive impairment. The SPMS cohort enrolled in the present study had a median EDSS score of 6.5 , which is higher than prior TBSS studies that were all primarily or solely composed of patients with RRMS with a median EDSS score of 3 or less. ${ }^{8,9,11}$ Generalized WM injury predominated in the present SPMS cohort with cognitive impairment, though preferential involvement of specific WM tracts was evident. Interestingly, prior studies of cognitive dysfunction in patients with RRMS primarily reported selective degradation of WM pathways associated with cognition but also showed widespread WM tract alteration. ${ }^{8,9,11}$ This inverted symmetry suggests that disease progression in cognitively impaired patients with MS, as demonstrated by EDSS score and MS subtype classification, is associated with evolution from a more tract specific imaging pattern to a more generalized imaging pattern.
Yu et $\mathrm{al}^{11}$ highlighted the significant correlations between reduced FA values and lower cognitive test scores in cognitively relevant tracts. However, significant correlations also occurred within all association fibers, commissural fibers, and projection fibers. This suggests that while WM abnormalities were principally tract selective, they appeared on a background of generalized WM alteration. Strikingly, the most extensively impaired WM regions in that study were the posterior thalamic radiation, CC, and sagittal stratum. Those results mirror our findings of diffuse WM dysfunction with residua of tract selectivity in the presence of greater disease severity.

We propose that even though WM dysfunction initially favors tracts associated with cognition, abnormalities become significantly more extensive and generalized as more domains are impaired and disease severity progresses. This suggests that cognitive impairment in patients with MS with progressive disease is a sequela of diffuse, nonspecific WM dysfunction. Such a pattern of WM alteration in turn raises the possibility that the underlying disease pathology itself is diffuse and nonspecific during the progressive phase, which aligns with the findings of a landmark postmortem study that investigated a variety of MS subtypes and found diffuse normal-appearing white matter injury to be a characteristic hallmark of progressive phase MS. ${ }^{33}$

Disease duration and disease severity were greater in our cognitively impaired patients than their nonimpaired counterparts, which suggests that cognitive dysfunction is associated with more advanced disease, though the findings did not reach statistical significance. Similarly, a recent study that also dichotomized patients according to performance on multiple cognitive tests demonstrated significantly higher EDSS scores in patients with MS with cognitive impairment. ${ }^{34}$ It has also been shown that patients with benign MS with cognitive impairment did not significantly differ from patients with SPMS with respect to normalized brain volume, global WML load, or GM mean diffusivity. ${ }^{35}$ In contrast, benign patients without cognitive impairment demonstrated significant differences.

Possible limitations of this study include sample size. The present cohort of patients with SPMS is relatively well populated $(n=45)$, but larger DTI studies of cognition in MS have been performed using different analytic approaches $(n=82) .{ }^{34}$ It is also possible that fatigue may have impacted the cognitive testing as it was not assessed. In addition, TBSS is a semi-automated technique that relies on a somewhat arbitrary FA threshold of 0.2 to define WM. In terms of cognition, we chose to solely focus on global cognitive impairment dichotomized based on the findings of a standard neuropsychological battery, instead of investigating individual subtests. This decision was motivated by the dearth of MS neuroimaging studies examining cognition as a multidomain entity, though this method is not without its own inherent limitations. Without the use of individual subtests, we were not able to fully establish a damage-response relationship where incremental changes in cognition can be correlated with incremental neuronal dysfunction.

Another potential limitation is our choice of SPMS as a disease subtype to investigate. RRMS is an earlier form of the disease and has been investigated much more frequently than SPMS. Our decision to examine patients with SPMS with cognitive impair- 
ment means that our results are therefore not directly comparable with previous DTI studies of MS and cognition..$^{8-11,13,34}$ However, our data do demonstrate structural differences between patients with SPMS with and without cognitive impairment and provide insight into the diffuse WM tract disturbances that occur with disease progression. Differences between impaired and nonimpaired patients with SPMS may also provide a potential surrogate marker for new therapeutic interventions to forestall the onset of cognitive impairment even in advanced disease.

\section{CONCLUSIONS}

This study sought to examine MR imaging correlates of multiple domain cognitive impairment in patients with SPMS with an emphasis on WM integrity. Impaired patients exhibited extensive WM dysfunction that severely disrupted every major tract in the brain while retaining predilection for pathways associated with cognition, such as the CC. These findings strongly suggest that cognitive impairment in patients with MS with advanced disease progression is predominantly the result of diffuse WM injury superimposed on a background of cognition-specific tract damage sustained during earlier stages of the disease. While impaired patients with SPMS also displayed significantly reduced global GM and WM volumes and significantly increased T2-weighted and T1-weighted WML loads, multivariate analysis revealed that only WM skeleton FA was a significant predictor of cognitive status.

Disclosures: Paul O'Connor-UNRELATED: Consultancy: Biogen Idec, Genzyme, Novartis, Roche, Teva, EMD Serono, Comments: Consulting fees for work related to MS consultations; Grants/Grants Pending: Biogen Idec, Roche, Sanofi-Genzyme, Comments: Funds for clinical trial not related to this work; Support for Travel to Meetings for the Study or Other Purposes: Travel to meeting in Lyons, Oct 2012, paid for by Novartis. Liesly Lee-UNRELATED: Consultancy: Consultation on advisory boards for Allergan, Biogen Canada, Serono Canada, Teva Neurosciences, BioMS, Schering, Bayer Canada, Novartis Canada, and Sanofi-Aventis. Anthony Feinstein-UNRELATED: Expert Testimony: MS Society of Canada; Payment for Lectures (including service on speakers' bureaus): Honoraria from Merck-Serono, Teva, Biogen, Novartis; Royalties: Books published by Cambridge University Press, Johns Hopkins University Press; Other: Stipend from CNN consultations. Richard Aviv—RELATED: Grant: *MS Society Physician Services Incorporated. *Money paid to institution.

\section{REFERENCES}

1. Rao SM, Leo GJ, Bernardin L, et al. Cognitive dysfunction in multiple sclerosis. I. Frequency, patterns, and prediction. Neurology 1991;41:685-91

2. Huijbregts SC, Kalkers NF, de Sonneville LM, et al. Differences in cognitive impairment of relapsing remitting, secondary, and primary progressive MS. Neurology 2004;63:335-39

3. Sperling RA, Guttmann CR, Hohol MJ, et al. Regional magnetic resonance imaging lesion burden and cognitive function in multiple sclerosis: a longitudinal study. Arch Neurol 2001;58:115-21

4. Benedict RH, Weinstock-Guttman B, Fishman I, et al. Prediction of neuropsychological impairment in multiple sclerosis: comparison of conventional magnetic resonance imaging measures of atrophy and lesion burden. Arch Neurol 2004;61:226-30

5. Houtchens MK, Benedict RH, Killiany R, et al. Thalamic atrophy and cognition in multiple sclerosis. Neurology 2007;69:1213-23

6. Papadopoulou A, Muller-Lenke N, Naegelin Y, et al. Contribution of cortical and white matter lesions to cognitive impairment in multiple sclerosis. Mult Scler 2013;19:1290-96

7. Rovaris M, Iannucci G, Falautano M, et al. Cognitive dysfunction in patients with mildly disabling relapsing-remitting multiple sclerosis: an exploratory study with diffusion tensor MR imaging. J Neurol Sci 2002;195:103-09

8. Dineen RA, Vilisaar J, Hlinka J, et al. Disconnection as a mechanism for cognitive dysfunction in multiple sclerosis. Brain 2009;132: 239-49

9. Roosendaal SD, Geurts JJ, Vrenken H, et al. Regional DTI differences in multiple sclerosis patients. Neuroimage 2009;44:1397-403

10. Warlop NP, Achten E, Fieremans E, et al. Transverse diffusivity of cerebral parenchyma predicts visual tracking performance in relapsing-remitting multiple sclerosis. Brain $\operatorname{Cog} n$ 2009;71:410-15

11. Yu HJ, Christodoulou C, Bhise V, et al. Multiple white matter tract abnormalities underlie cognitive impairment in RRMS. Neuroimage 2012;59:3713-22

12. Filippi M, Rocca MA, Benedict RH, et al. The contribution of MRI in assessing cognitive impairment in multiple sclerosis. Neurology 2010;75:2121-28

13. Hawellek DJ, Hipp JF, Lewis CM, et al. Increased functional connectivity indicates the severity of cognitive impairment in multiple sclerosis. Proc Natl Acad Sci USA 2011;108:19066-71

14. Hulst HE, Steenwijk MD, Versteeg A, et al. Cognitive impairment in MS: impact of white matter integrity, gray matter volume, and lesions. Neurology 2013;80:1025-32

15. Lucchinetti CF, Popescu BF, Bunyan RF, et al. Inflammatory cortical demyelination in early multiple sclerosis. N Engl J Med 2011;365: 2188-97

16. Aviv RI, Francis PL, Tenenbein R, et al. Decreased frontal lobe gray matter perfusion in cognitively impaired patients with secondaryprogressive multiple sclerosis detected by the bookend technique. AJNR Am J Neuroradiol 2012;33:1779-85

17. Francis PL, Jakubovic R, O'Connor $\mathrm{P}$, et al. Robust perfusion deficits in cognitively impaired patients with secondary-progressive multiple sclerosis. AJNR Am J Neuroradiol 2013;34:62-67

18. Khalil M, Enzinger C, Langkammer C, et al. Cognitive impairment in relation to MRI metrics in patients with clinically isolated syndrome. Mult Scler 2011;17:173-80

19. Smith SM, Jenkinson M, Johansen-Berg H, et al. Tract-based spatial statistics: voxelwise analysis of multi-subject diffusion data. Neuroimage 2006;31:1487-505

20. Compston A, Coles A. Multiple sclerosis. Lancet 2008;372:1502-17

21. Kurtzke JF. Rating neurologic impairment in multiple sclerosis: an expanded disability status scale (EDSS). Neurology 1983;33:1444-52

22. Benedict RH, Fischer JS, Archibald CJ, et al. Minimal neuropsychological assessment of MS patients: a consensus approach. Clin Neuropsychol 2002;16:381-97

23. Benedict RH, Cookfair D, Gavett R, et al. Validity of the minimal assessment of cognitive function in multiple sclerosis (MACFIMS). J Int Neuropsychol Soc 2006;12:549-58

24. Smith SM, Jenkinson M, Woolrich MW, et al. Advances in functional and structural MR image analysis and implementation as FSL. Neuroimage 2004;23(suppl 1):S208-19

25. Smith SM, Johansen-Berg H, Jenkinson M, et al. Acquisition and voxelwise analysis of multi-subject diffusion data with tract-based spatial statistics. Nat Protoc 2007;2:499-503

26. Nichols TE, Holmes AP. Nonparametric permutation tests for functional neuroimaging: a primer with examples. Hum Brain Mapp 2002;15:1-25

27. Smith SM, Nichols TE. Threshold-free cluster enhancement: addressing problems of smoothing, threshold dependence and localisation in cluster inference. Neuroimage 2009;44:83-98

28. Mori S, Oishi K, Jiang H, et al. Stereotaxic white matter atlas based on diffusion tensor imaging in an ICBM template. Neuroimage 2008;40:570-82

29. Hines M, Chiu L, McAdams LA, et al. Cognition and the corpus callosum: verbal fluency, visuospatial ability, and language lateralization related to midsagittal surface areas of callosal subregions. Behav Neurosci 1992;106:3-14

30. Chiang MC, Barysheva M, Shattuck DW, et al. Genetics of brain fiber architecture and intellectual performance. J Neurosci 2009;29:2212-24

31. Cantero JL, Atienza M, Gomez-Herrero G, et al. Functional integrity of thalamocortical circuits differentiates normal aging from mild cognitive impairment. Hum Brain Mapp 2009;30:3944-57 
32. Cho H, Yang DW, Shon YM, et al. Abnormal integrity of corticocortical tracts in mild cognitive impairment: a diffusion tensor imaging study. J Korean Med Sci 2008;23:477-83

33. Kutzelnigg A, Lucchinetti CF, Stadelmann C, et al. Cortical demyelination and diffuse white matter injury in multiple sclerosis. Brain 2005; 128:2705-12
34. Mesaros S, Rocca MA, Kacar K, et al. Diffusion tensor MRI tractography and cognitive impairment in multiple sclerosis. Neurology 2012;78:969-75

35. Rovaris M, Riccitelli G, Judica E, et al. Cognitive impairment and structural brain damage in benign multiple sclerosis. Neurology 2008;71:1521-26 